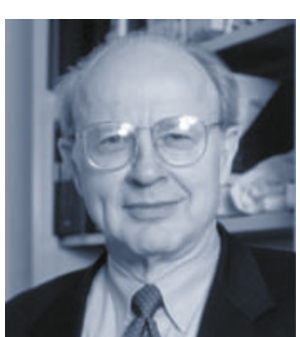

Robert Roeder

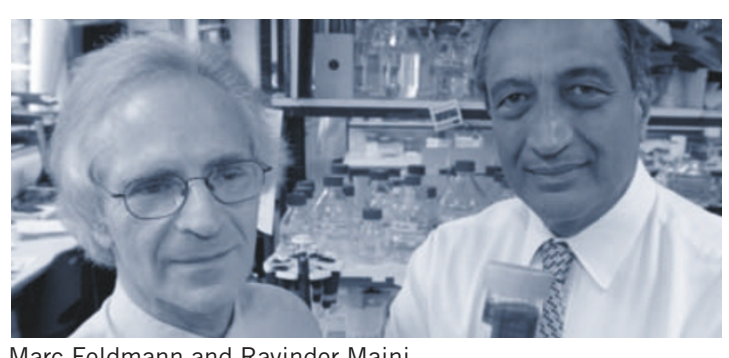

Marc Feldmann and Ravinder Maini

\title{
Lasker goes transcriptional
}

The Lasker prize was established almost 60 years ago, two years after the discovery that DNA constitutes the genetic material, and may be relatively young when compared with the godfather of all prizes, the Nobel. Nevertheless, it has garnered a reputation for not lagging far behind, built on a remarkable list of past awardees.

Although focused on awarding recognition to medical research that has enabled the understanding, prevention and treatment of disease, the Lasker juries have always made a point of honouring both basic and clinical research. The philosophy that true biological understanding, and consequently medical progress, relies on a detailed appreciation of the molecular principles underlying complex biological processes is, of course, what spurs on most researchers in molecular biology. Nature Cell Biology has increasingly moved to incorporate molecular biology into its editorial purview, reflecting the considerable advances in our molecular understanding of cell biology (also see September's editorial (DOI: 10.1038/ncb0903-763).

No area is more at the core of molecular biology than gene transcription, and no person is more at the core of transcription than this year's Lasker awardee for basic medical research, Robert Roeder of the Rockefeller University (NY). The principal advances of Roeder cited by the Lasker jury are his discovery, functional charcaterisation and purification of the three eukaryotic RNA polymerases, leading to the establishment of in vitro transcription protocols. Joseph Goldstein, chair of the Lasker awards jury, comments that this latter accomplishment "opened the field of RNA transcription in much the same way that Arthur Kornberg opened the field of DNA replication by synthesizing DNA". It is equally fair to say that Roeder's three DEAE-dextran column elution peaks of 1969 (designated by him RNA polymerase I, II and III) were as important a milestone in transcription research as was Jacob and Monod's work in prokaryotes a decade earlier. Nevertheless, his outstanding contributions to the field continued with a dissection of the complex set of accessory general initiation factors, leading to a detailed definition of pre-initiation complex (PIC) assembly for the three classes of genes over the following two decades. Roeder continued to identify a number of important sequence-specific transcription factors and co-activator/mediator complexes.

Gene transcription is a key downstream target for most signalling pathways and is one of the central ways in which the expression of gene products is regulated. In addition, transcriptional regulation is integrated with DNA repair, cell cycle checkpoints and chromatin remodelling. The precise spatio-temporal regulation of specific genes underlies most physiological responses, such as development, cell growth and differentiation, and the immune response, as so strikingly demonstrated these days by microarray data and in situ hybridization. Almost every process derailed in cancer ultimately leads to altered gene expression profiles. As a result, it is maybe not surprising that the complexity of the eukaryotic transcriptional apparatus has grown so dramatically in several waves of discovery (RNA polymerases, basal transcription factors, specific transcription factors, coactivators/repressors and chromatin remodelling factors) to form the highly regulatable and specific multisubunit machines we appreciate today.

The clinical research award highlights a classic, and still all too rare, bench-to-bedside success: Marc Feldmann and Ravinder Maini of the Imperial College School of Medicine (London) are honoured for establishing anti-tumour necrosis factor (TNF)- $\alpha$ therapy as an effective treatment for autoimmune diseases such as rheumatoid arthritis and Crohn disease, with three licensed drugs currently available. This long-term collaboration between the basic immunologist Feldmann and the clinical rheumatologist Maini saw them delineate the TNF cascade of inflammatory cytokines in rheumatism, test antibody inhibition of TNF in animal models and translate the concept into the clinic. As such, this highly successful collaboration should inspire as a paradigm for mining the considerable clinical potential of basic cell biology. 